\title{
Accurate analytic approximation for the Chapman grazing incidence function
}

\author{
Dmytro Vasylyev ${ }^{*}$ (1)
}

\begin{abstract}
A new analytical approximation for the Chapman mapping integral, $\mathrm{Ch}$, for exponential atmospheres is proposed. This formulation is based on the derived relation of the Chapman function to several classes of the incomplete Bessel functions. Application of the uniform asymptotic expansion to the incomplete Bessel functions allowed us to establish the precise analytical approximation to $\mathrm{Ch}$, which outperforms established analytical results. In this way the resource consuming numerical integration can be replaced by the derived approximation with higher accuracy. The obtained results are useful for various branches of atmospheric physics such as the calculations of optical depths in exponential atmospheres at large grazing angles, physical and chemical aeronomy, atmospheric optics, ionospheric modeling, and radiative transfer theory.
\end{abstract}

Keywords: Chapman mapping function, Exponential atmosphere, Incomplete cylindrical function, Optical thickness, Optical air mass, Atmospheric aeronomy

\section{Introduction}

In modeling of atmospheres their chemical constituents are often assumed to be distributed according to the hydrostatic equilibrium within the column extending from a point of interest towards the sun. This columnar content can be conveniently mapped to the reference value via the Chapman grazing incidence function Chapman (1931). The reference value corresponds to the vertical column, i.e., to the situation when the sun is at the zenith of the point of interest. As an approximation to the hydrostatic equation the distribution of atmospheric constituents is assumed to follow the exponential distribution within the vertical column. Being originally introduced for the description of energy deposition in the ionosphere due to the absorption of solar radiation, the Chapman function is now an important mathematical object in other branches of the atmospheric physics, such as radiative transfer theory (Dahlback and Stamnes 1991), chemical and physical aeronomy (Brasseur and

\footnotetext{
*Correspondence: dmytro.vasylyev@dlr.de

Deutsches Zentrum für Luft- und Raumfahrt, Institut für Solar-

Terrestrische Physik, Neustrelitz, Germany
}

Solomon 2005; Schunk and Nagy 2009), description of light scattering in planetary atmospheres (Wallach and Hapke 1985). The function is also used in modeling of ionospheric D-layer (Friedrich and Torkar 2001), in calculations of atmospheric transparency (Green et al. 1964), optical air masses (Kocifaj 1996; Rapp-Arrarás and Domingo-Santos 2011), cosmic ray ionization rates (Velinov 1974), or for air shower simulations (Extensive and Showers 2010), to name just a few.

In general case the evaluation of the Chapman function requires numerical integration, which, in turn, might require considerable computational resources in complex physical models of exponential atmospheres. This is the reason why many attempts have been made in order to provide simple analytic approximative expressions for the Chapman integral. The most notorious approximations of the Chapman function have been collected in the $\mathrm{D}$. Huestis work (Huestis 2001). This work is also an excellent source on numerical and asymptotical evaluation of the various integral representations of this mapping function.

The aim of the present article is to draw the reader attention to the less known interrelation of the Chapman 
function and the incomplete cylindrical functions (Agrest and Maximov 1971). Based on this observation we obtain new asymptotic representations of the Chapman function, which approximate the exact integral expressions with the high order of accuracy. One of the derived approximations yields better approximation of the exact Chapman integral than other analytic approximations found in the literature. We hope that the obtained result would be useful for physical models of ionospheres, theories of optical propagation in the atmospheres, radiative transfer theories, and in remote sensing applications

The article is organized as follows. In section "Definitions and current analytic approximations" we summarize the basic definitions and properties of the Chapman function and give an overview of its existing analytic approximations. In section "Derivation of new analytic approximations" we derive the relationship of the Chapman integral with several classes of the incomplete cylindrical functions. Based on these relationships and using the uniform asymptotic expansions of the incomplete Macdonald integrals we derive analytic approximations of the Chapman mapping function. The methodological aspects of comparison of different analytic approximations are discussed in "Methodology of comparison". The performance of the obtained approximations is discussed in section "Results of comparison and discussion" and is compared with other approximations found in the literature. The obtained results are summarized in "Conclusions".

\section{Definitions and current analytic approximations}

The derivation of Chapman mapping function relies on several simplifications concerning the properties and geometry of atmospheric layers. Firstly, the spherical symmetry of the atmospheric shells is assumed. Secondly, the hydrostatic equilibrium within the atmospheric shells is expected, meaning that in a state of rest, the atmospheric pressure at specified height must support the weight of the gas above it. Additionally to this the temperature and the gravitational acceleration are supposed not to vary with the altitude. This restriction results in exponential distribution of an ideal gas in hydrostatic equilibrium. Finally, the bending of solar rays due to atmospheric refraction is neglected.

Under specified restrictions the classical representation of the Chapman function as derived in Chapman (1931) reads as

$$
\operatorname{Ch}(X, \zeta)=X \sin \zeta \int_{0}^{\zeta} \exp [X(1-\sin \zeta \csc t)] \csc ^{2} t \mathrm{~d} t,(1)
$$

where $\zeta$ is the solar zenith angle at the point of interest and

$$
X=\frac{L}{H}
$$

is the dimensionless ratio of the spatial coordinate $L$ measured from the center of the Earth to the scale height $H$ of particular atmospheric gas. The scale height characterizes the exponential decay of the atmospheric constituent density with the height. For an ideal gas this quantity is expressed through the Boltzman's constant $k$, the temperature $T$, the mean constituent mass $m$, and the acceleration due to gravity $g$ as

$$
H=k T / m g \text {. }
$$

If the spherical earth model is under consideration, we have $L=R_{\oplus}+z$ with $R_{\oplus}$ being the earth radius and $z$ being the hight of the point of interest. The derivation of Eq. (1), based on geometrical considerations, is summarized in Swider (1964).

To avoid numerical integration of Eq. (1) several analytic approximations have been proposed in the literature. These approximations are based either on asymptotic evaluation of the integral or on semi-empirical considerations. Table 1 summarizes the most widely used approximative Chapman mapping functions. Most of the expressions are given in terms of the complimentary error function

$$
\operatorname{erfc}(x)=\frac{2}{\sqrt{\pi}} \int_{x}^{\infty} e^{-t^{2}} \mathrm{~d} t
$$

which in contrast to Eq. (1) being a single argument function is properly tabulated and approximated, cf. Abramowitz and Stegun (1972). The formulas in Table 1 are given for the solar zenith angle $0 \leq \zeta \leq \pi / 2$ and can be extended to larger zenith angles via the relationship (Chapman 1953; Titheridge 1988):

$$
\begin{aligned}
\operatorname{Ch}(X, \zeta)= & 2 \exp [X(1-\sin \zeta)] \operatorname{Ch}(X \sin \zeta, \pi / 2) \\
& -\operatorname{Ch}(X, \pi-\zeta), \quad \zeta>\pi / 2 .
\end{aligned}
$$

Here

$$
\operatorname{Ch}(X \sin \zeta, \pi / 2)=X \sin \zeta e^{X \sin \zeta} K_{1}(X \sin \zeta)
$$

and $K_{1}$ is the modified Bessel function of the second kind of the first order (Macdonald function), cf. Abramowitz and Stegun (1972). At the limiting case of $\zeta=\pi / 2$ we obtain the Chapman function at the horizon for the point of interest:

$$
\operatorname{Ch}(X, \pi / 2)=X e^{X} K_{1}(X) .
$$

Due to Eq. (3) we restrict our attention in the following to zenith angles in the range $\zeta \in[0, \pi / 2]$. 
Table 1 Analytical approximations of the Chapman function (in chronological order). The solar zenith angle $\zeta$ is given in radian units

\begin{tabular}{|c|c|c|}
\hline Index & Reference & Approximation of the Chapman function \\
\hline (a) & Green et al. (1964); Green and Barnum (1963) & $\begin{array}{l}\operatorname{Ch}(X, \zeta) \approx \exp \left[\frac{\frac{1}{2} \zeta^{2}}{1-0.115 \zeta^{2}-\alpha(X) \zeta^{4}}\right] \\
\alpha(X)=\frac{16}{\pi^{4}}\left\{1-(\pi / 2)^{2}[0.115+1 / \ln (X \pi / 2)]\right\}\end{array}$ \\
\hline (b) & Fitzmaurice (1964) & $\operatorname{ch}(X, \zeta) \approx \sqrt{\frac{\pi x}{2}} \operatorname{erfc}\left(\sqrt{\frac{X}{2}} \cos \zeta\right) \exp \left(\frac{x}{2} \cos ^{2} \zeta\right)$ \\
\hline (c) & Swider (1964) & $\begin{array}{l}\operatorname{Ch}(X, \zeta) \approx-X \cos \zeta+\sqrt{1+X(1+\sin \zeta)}\{\sqrt{X(1-\sin \zeta)} \\
\left.+\frac{\sqrt{\pi}}{2} \operatorname{erfc}[\sqrt{X(1-\sin \zeta)}] \exp [X(1-\sin \zeta)]\right\}\end{array}$ \\
\hline (d) & Titheridge $(1988,2000)$ & $\begin{array}{l}\operatorname{Ch}(X, \zeta) \approx \sec (\zeta-d), \quad d=3.88 X^{-1.143}[\sec (A \zeta)-0.834] \\
A=1.0123-1.454 X^{-1 / 2}\end{array}$ \\
\hline (e) & Kocifaj (1996); Schüler (2012) & $\begin{array}{l}\operatorname{Ch}(X, \zeta) \approx \frac{1}{2}\left[\cos \zeta+\sqrt{\frac{\pi X}{2}} \operatorname{erfc}\left(\sqrt{\frac{X}{2}} \cos \zeta\right) \exp \left(\frac{x}{2} \cos ^{2} \zeta\right)\right. \\
\left.\times\left(\frac{1}{x}+1+\sin ^{2} \zeta\right)\right]\end{array}$ \\
\hline$(f)$ & Huestis (2001) & $\operatorname{Ch}(X, \zeta) \approx \sqrt{\frac{\pi X}{(1+\sin \zeta)}} \operatorname{erfc}[\sqrt{X(1-\sin \zeta)}] \exp [X(1-\sin \zeta)]$ \\
\hline
\end{tabular}

\section{Derivation of new analytic approximations} Relation to incomplete cylindrical functions

Here we show the relationship of the Chapman function to several families of the incomplete cylindrical functions. To start with, we change the integration variable $t$ in Eq. (1) according to $s=\csc (t)$. In this way we arrive at the alternative integral representation of the Chapman function

$$
\operatorname{Ch}(X, \zeta)=X \sin \zeta e^{X} \int_{\cosh \beta}^{\infty} \exp (-X s \sin \zeta) s\left(s^{2}-1\right)^{-1 / 2} \mathrm{~d} s,
$$

where

$$
\beta=\ln \frac{1+\cos \zeta}{\sin \zeta}=\ln \cot \frac{\zeta}{2}=\cosh ^{-1}(\csc \zeta) .
$$

Integrating Eq. (6) by parts we obtain

$$
\begin{aligned}
\operatorname{Ch}(X, \zeta)= & -X \sin \zeta \sinh \beta+X^{2} \sin ^{2} \zeta e^{X} \\
& \int_{\cosh \beta}^{\infty} \exp (-s X \sin \zeta) \sqrt{s^{2}-1} \mathrm{~d} s
\end{aligned}
$$

From this formula it is easy to derive the result (5) for $\zeta=\pi / 2$, i.e., $\cosh \beta=1$, by using the integral representation of the Macdonald function (Abramowitz and Stegun 1972, Eq. 9.6.23)

$$
K_{n}(x)=\frac{\sqrt{\pi}\left(\frac{1}{2} x\right)^{n}}{\Gamma\left(n+\frac{1}{2}\right)} \int_{1}^{\infty} \exp (-s x)\left(s^{2}-1\right)^{n-\frac{1}{2}} \mathrm{~d} s,
$$

where $\Gamma(v)$ is the Gamma function.

In the view of Eq. (9) the Chapman function (8) can be rewritten as

$$
\begin{aligned}
\mathrm{Ch}(X, \zeta)= & -X \sin \zeta \sinh \beta+X \sin \zeta e^{X} \\
& {\left[K_{1}(X \sin \zeta)-L_{1}(X \sin \zeta, \cosh \beta)\right] }
\end{aligned}
$$

with

$$
L_{n}(x, y)=\frac{\sqrt{\pi}\left(\frac{1}{2} x\right)^{n}}{\Gamma\left(n+\frac{1}{2}\right)} \int_{1}^{y} \exp (-s x)\left(s^{2}-1\right)^{n-\frac{1}{2}} \mathrm{~d} s
$$

being the incomplete Macdonald function in the form introduced in Agrest and Maximov (1971). The nomenclature "incomplete cylindrical function" or "incomplete Bessel function" becomes clear from this definition as only in the limit $y \rightarrow \infty$ the function (11) becomes the "complete" Macdonald function (9). 
In order to relate the Chapman function to other types of incomplete cylindrical functions we perform the variable change in (8) according to $s=\cosh \theta$ and obtain
We also note that the limiting case (5) for $\zeta=\pi / 2$ is obtained from (15) by observing that $K_{n}(x, 0)=K_{n}(x)^{1}$ and with the use of the well-known recurrence relations

$$
\operatorname{Ch}(X, \zeta)=-X \sin \zeta \sinh \beta+X^{2} \sin ^{2} \zeta e^{X} \int_{\beta}^{\infty} \exp (-X \sin \zeta \cosh \theta) \sinh ^{2} \theta \mathrm{d} \theta
$$

This expression allows one to express the Chapman function through the incomplete Macdonald functions

$$
K_{n}(x, y)=\int_{y}^{\infty} e^{-x \cosh \theta} \cosh (n \theta) \mathrm{d} \theta
$$

as defined by Jones (2007) or

$$
\tilde{K}_{n}(x, y)=\int_{1}^{\infty} e^{-(x t+y / t)} t^{n-1} \mathrm{~d} t
$$

as defined by Terras (1981). Namely, using the definition for $K_{n}(x, y)$ the Chapman function can be represented as for the Bessel functions.

The recently discovered relationship of the Chapman function to the solution of an inhomogeneous modified Bessel's equation (Huestis 2001) yields the following representation of this mapping function:

$$
\begin{aligned}
\operatorname{Ch}(X, \zeta)= & X \sin \zeta \cos \zeta e^{X} \int_{X}^{\infty} e^{-t} \\
& {\left[I_{1}(X \sin \zeta) K_{0}(t \sin \zeta)+K_{1}(X \sin \zeta) I_{0}(t \sin \zeta)\right] \mathrm{d} t, }
\end{aligned}
$$

where $I_{n}(x)$ are the modified Bessel functions of $n$th order. The advantage of this integral representation is that the Champan function in this formulation is stable

$$
\operatorname{Ch}(X, \zeta)=-X \sin \zeta \sinh \beta+\frac{1}{2} X^{2} \sin ^{2} \zeta e^{X}\left[K_{2}(X \sin \zeta, \beta)-K_{0}(X \sin \zeta, \beta)\right] .
$$

The corresponding relationship between the Chapman function and the incomplete cylindrical function (14) is easily obtained from (15) by observing the obvious relation

$$
K_{n}(x, y)=\frac{1}{2}\left[e^{n y} \tilde{K}_{n}\left(\frac{x}{2} e^{y}, \frac{x}{2} e^{-y}\right)+e^{-n y} \tilde{K}_{-n}\left(\frac{x}{2} e^{y}, \frac{x}{2} e^{-y}\right)\right] .
$$

The recurrence relations also allow to establish the relationship between the incomplete Bessel functions in form of Agrest and Maximov, Jones, and Terras. For example, we have in our case of interest the following relations

$$
L_{0}(x, \cosh y)=K_{0}(x)-K_{0}(x, y),
$$

and

$$
L_{1}(x, \cosh y)=K_{1}(x)-K_{1}(x, y)+\tanh y \frac{\partial}{\partial y} K_{1}(x, y) .
$$

for all values of $X$ and $\zeta$ as has been shown by Huestis (2001). The integrals in Eq. (19)

$$
\mathcal{I}_{1}(X, \zeta)=\int_{X}^{\infty} e^{-t} I_{0}(t \sin \zeta) \mathrm{d} t, \quad \mathcal{I}_{2}(X, \zeta)=\int_{X}^{\infty} e^{-t} K_{0}(t \sin \zeta) \mathrm{d} t
$$

cannot be analytically integrated in general case except for $X=0$. One can show that these integrals possess a weak singularity for $t \rightarrow \infty, \zeta=\pi / 2$ while $\mathcal{I}_{2}$ possesses an integrable singularity for $t \rightarrow 0, \zeta \rightarrow 0$.

Equation (19) allows one to establish the relation of the Chapman function and another class of the incomplete cylindrical functions known as the incomplete LipschitzHankel integrals (Agrest and Maximov 1971). Namely, the integrals (20) can be expressed through the incomplete Lipschitz-Hankel integrals (Agrest and Maximov 1971)

\footnotetext{
${ }^{1}$ Alternatively the property

$$
K_{n}(x)=\frac{1}{2}\left[\tilde{K}_{n}(x / 2, x / 2)+\tilde{K}_{-n}(x / 2, x / 2)\right]
$$

is applied if the Terras' representation for incomplete Bessel functions is used.
} 


$$
I e_{0}(x, y)=\int_{0}^{y} e^{-x t} I_{0}(t) \mathrm{d} t, \quad K e_{0}(x, y)=\int_{0}^{y} e^{-x t} K_{0}(t) \mathrm{d} t
$$

as

$$
\mathcal{I}_{1}(X, \zeta)=\sec \zeta-\csc \zeta I e_{0}(\csc \zeta, X \sin \zeta), \quad 0 \leq \zeta<\pi / 2
$$

$\mathcal{I}_{2}(X, \zeta)=\beta \sec \zeta-\csc \zeta K e_{0}(\csc \zeta, X \sin \zeta), \quad 0 \leq \zeta<\pi / 2$.

Here the parameter $\beta$ is given by Eq. (7). Substituting Eqs. (22), (23) in Eq. (19) we obtain

$$
\begin{aligned}
\operatorname{Ch}(X, \zeta)= & X \sin \zeta e^{X}\left\{I_{1}(X \sin \zeta) \beta+K_{1}(X \sin \zeta)\right. \\
& \left.-\cot \zeta\left[I_{1}(X \sin \zeta) K e_{0}(\csc \zeta, X \sin \zeta)+K_{1}(X \sin \zeta) I e_{0}(\csc \zeta, X \sin \zeta)\right]\right\}
\end{aligned}
$$

This representation reduces $\mathrm{Ch}(X, \pi / 2)$ directly to the limiting case (5).

Using Eq. (19) we now derive the relationship of the Chapman function to the incomplete Bessel functions (13). We start with the result obtained in Ref. Agrest and Maximov (1971):

$$
L_{0}(x, \cosh y)=y I_{0}(x)+\sinh y \int_{0}^{x}\left[K_{0}(x) I_{0}(t) e^{-t \cosh y}-I_{0}(x) K_{0}(t) e^{-t \cosh y}\right] \mathrm{d} t,
$$

where the function $L_{0}$ is defined in Eq. (11). Differentiating both sides of Eq. (25) with respect to $x$ and using the relations $\mathrm{d} I_{0}(x) / \mathrm{d} x=I_{1}(x)$ and $\mathrm{d} K_{0}(x) / \mathrm{d} x=-K_{1}(x)$ we obtain

$$
\frac{\mathrm{d}}{\mathrm{d} x} L_{0}(x, \cosh y)=y I_{1}(x)-\sinh y \int_{0}^{x}\left[K_{1}(x) I_{0}(t) e^{-t \cosh y}+I_{1}(x) K_{0}(t) e^{-t \cosh y}\right] \mathrm{d} t .
$$

This equation allows one to rewrite the Chapman function (19) as

$$
\operatorname{Ch}(X, \zeta)=\frac{1}{2} X \sin \zeta e^{X}\left[K_{1}(X \sin \zeta, \beta)+K_{-1}(X \sin \zeta, \beta)\right]=X \sin \zeta e^{X} K_{1}(X \sin \zeta, \beta),
$$

where the property $K_{-n}(x, y)=K_{n}(x, y)$ has been used. This is alternative to Eq. (15) representation of the Chapman function in terms of the incomplete Macdonald functions (13).

To conclude this section we relate the Chapman function with another class of the incomplete cylindrical 


$$
I e_{0}(\csc \zeta, X \sin \zeta)=\tan \zeta\left\{2 e^{-2 X \sin ^{2} \frac{\zeta}{2}} \widetilde{Q}_{0}\left(X \sin ^{2} \frac{\zeta}{2}, X \sin \zeta\right)-\left[1-e^{-X} I_{0}(X \sin \zeta)\right]\right\}
$$

The incomplete integral $K e_{0}(x, y)$ can be expressed in terms of $I e_{0}(x, y)$ as shown in Ref. Agrest and Maximov (1971) as

$$
\begin{aligned}
K e_{0}(\csc \zeta, X \sin \zeta)= & \frac{K_{0}(X \sin \zeta)}{I_{0}(X \sin \zeta)} I e_{0}(\csc \zeta, X \sin \zeta) \\
& +\tan \zeta \frac{1}{I_{0}(X \sin \zeta)}\left[\beta I_{0}(X \sin \zeta)-L_{0}(X \sin \zeta, \cosh \beta)\right]
\end{aligned}
$$

values of the scale parameter $X$, which is not of interest in atmospheric physics applications, is based on Eq. (31) and is considered in the Appendix A. where $L_{n}(x, y)$ is given by (11). Combining Eq. (29) with (30) and substituting them in (24) we obtain

$$
\begin{aligned}
\operatorname{Ch}(X, \zeta)= & X \sin \zeta e^{X}\left(K_{1}(X \sin \zeta)+\frac{I_{1}(X \sin \zeta)}{I_{0}(X \sin \zeta)}\left[K_{0}(X \sin \zeta)-K_{0}(X \sin \zeta, \beta)\right]\right. \\
& -\left[K_{1}(X \sin \zeta)-\frac{I_{1}(X \sin \zeta)}{I_{0}(X \sin \zeta)} K_{0}(X \sin \zeta)\right] \\
& \left.\times\left\{2 e^{-2 X \sin ^{2} \frac{\zeta}{2}} \widetilde{Q}_{0}\left(X \sin ^{2} \frac{\zeta}{2}, X \sin \zeta\right)-\left[1-e^{-X} I_{0}(X \sin \zeta)\right]\right\}\right)
\end{aligned}
$$

where we have used Eq. (17) for the incomplete Bessel function $L_{0}(x, y)$. The obtained expression is rather complex to be applied in practice. Its derivation serves merely for sake of completeness of the relations between the Chapman function and the incomplete cylindrical functions of different kinds. However, it appears that (31) becomes useful for the analytic approximation of the Chapman function when the scale parameter is small.

\section{Analytic approximations for the mapping function}

In the previous section we have obtained several formulas that relate the Chapman mapping function (1) to the incomplete cylindrical functions of various types. Specifically, Eq. (10) relates the Chapman integral to the incomplete Macdonald function $L_{n}(x, y)$ of the Agrest and Maksimov type, whereas Eqs. (15) and (27) express the former in terms of the incomplete Macdonald function $K_{n}(x, y)$ of the Jones type or alternatively in terms of the Terras incomplete Macdonald function $\tilde{K}_{n}(x, v)$. Finally, Eq. (24) is the representation in terms of the incomplete Lipschitz-Hankel integrals $I e_{n}(x, y), K e_{n}(x, y)$ and can be also rewritten in terms of the incomplete Weber integral $\widetilde{Q}_{n}(x, y)$ and the incomplete Macdonald function as given in Eq. (31). Since there are relationships among these various types of incomplete Bessel functions as discussed in the previous section, we consider the analytic approximation of the Chapman function using Eqs. (15) and (27) written in terms of $K_{n}(x, y)$. The special case of small dictated by suitability of its integral representation (13) for uniform asymptotic expansions. We refer to the work (Jones 2007) for details in derivation of the expansion formula and give here the lowest terms in the asymptotic expansion of $K_{n}(x, y)$

$$
\begin{aligned}
K_{n}(X \sin \zeta, \beta) \approx & \left(\frac{\pi}{2 X \sin \zeta}\right)^{1 / 2}\left(\alpha_{0}+\frac{\alpha_{1}}{X \sin \zeta}\right) e^{-X \sin \zeta} \\
& \operatorname{erfc}\left(\sqrt{2 X \sin \zeta} \sinh \frac{\beta}{2}\right) \\
& +\left(\frac{\beta_{0}}{X \sin \zeta}+\frac{\beta_{1}}{X^{2} \sin ^{2} \zeta}\right) e^{-X}
\end{aligned}
$$

Here $\beta$ is given in Eq. (7) and

$$
\begin{aligned}
\alpha_{0}= & 1, \quad \alpha_{1}=\frac{1}{8}\left(4 n^{2}-1\right), \\
\beta_{0}= & \frac{\cosh n \beta}{\sinh \beta}-\frac{1}{2 \sinh \frac{\beta}{2}}, \\
\beta_{1}= & \frac{n \sinh n \beta}{\sinh ^{2} \beta}-\frac{\cosh n \beta \cosh \beta}{\sinh ^{3} \beta} \\
& +\frac{1}{8 \sinh ^{3} \frac{\beta}{2}}-\frac{4 n^{2}-1}{16 \sinh \frac{\beta}{2}} .
\end{aligned}
$$


This uniform asymptotic expansion of the incomplete Bessel function allows us to obtain the new analytic approximations of the Chapman integral, which are the main results of the present article. Substituting (32) in (15) yields

$$
\begin{aligned}
\operatorname{Ch}(X, \zeta) \approx & \sqrt{\frac{\pi}{2}} \sqrt{X \sin \zeta} e^{X(1-\sin \zeta)} \\
& \operatorname{erfc}\left(\sqrt{2 X \sin \zeta} \sinh \frac{\beta}{2}\right) \\
& +\operatorname{coth} \beta-\frac{1}{2 \sinh \frac{\beta}{2}} .
\end{aligned}
$$

Inserting (32) in (27) we obtain

$$
\begin{aligned}
\operatorname{Ch}(X, \zeta) \approx & \sqrt{\frac{\pi}{2}} \sqrt{X \sin \zeta}\left(1+\frac{3}{8} \frac{1}{X \sin \zeta}\right) e^{X(1-\sin \zeta)} \\
& \operatorname{erfc}\left(\sqrt{2 X \sin \zeta} \sinh \frac{\beta}{2}\right) \\
& +\operatorname{coth} \beta-\frac{1}{2 \sinh \frac{\beta}{2}}-\frac{1}{X \sin \zeta} \\
& \left(\frac{1}{\sinh ^{3} \beta}-\frac{1}{8 \sinh ^{3} \frac{\beta}{2}}+\frac{3}{16 \sinh \frac{\beta}{2}}\right) \\
\beta= & \cosh ^{-1}(\csc \zeta) .
\end{aligned}
$$

We see that Eq. (32) is more suitable for deriving of asymptotic expansion of the Chapman function since it preserves the order of expansion of the incomplete Bessel function (27) in contrast to the definition (15).

Inspection of Eq. (36) shows that the first term on the r.h.s resembles the asymptotic expansion of the Macdonald function $K_{1}(X \sin \zeta)$ with the complementary error function. This observation shows the similarity of the obtained asymptotic expansion formula (36) with the large$\zeta$ expansion of Chapman, cf. Eq.(38) in his original article (Chapman 1931). If one performs the expansion of the Macdonald function up to the second term in the first summand of the Chapman asymptotic expression, one obtains exactly the first term of Eq. (36). With this respect it is worth to note that Chapman considered the asymptotic of small and large zenith angles separately. Moreover, Chapman's original asymptotic expansions are complex polynomial series with poor convergence, the issue corrected in Ref. Huestis (2001). Equation (36) originates from uniform

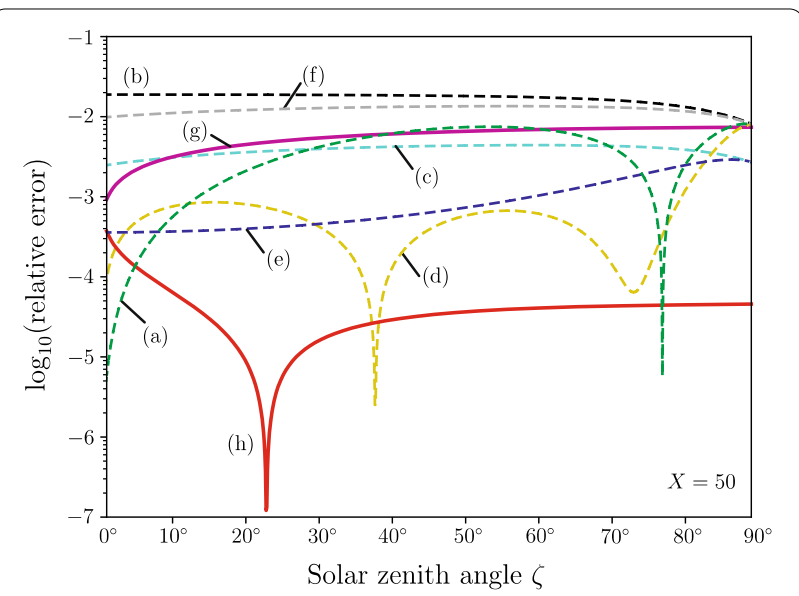

Fig. 1 Accuracy of analytical approximations of the Chapman function as a function of solar zenith angle $\zeta$ for scale parameter $X=50$. The dashed lines show the formulas found in the literature and solid lines correspond to approximations introduced in the present article (labeling in chronological order, see Table 1): a Green \& Barnum approximation (Green and Barnum 1963), b Fitzmaurice approximation (Fitzmaurice 1964), c Swider approximation (Swider 1964), d Titheridge empirical formula (Titheridge 1988), e Kocifaj formula (Kocifaj 1996), f Huestis asymptotics, 1 term (Huestis 2001), $\mathbf{g}$ present work, Eq. (35), h present work, Eq. (36)

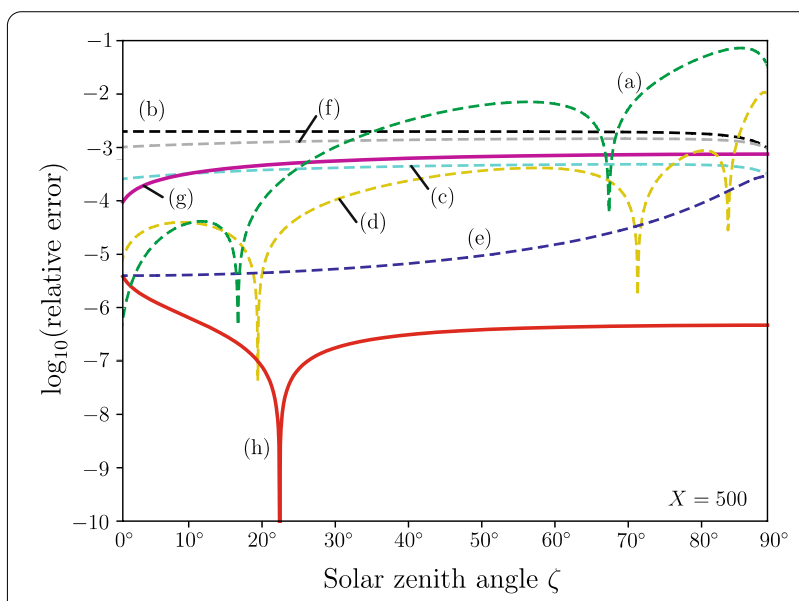

Fig. 2 Accuracy of analytical approximations of the Chapman function as a function of solar zenith angle $\zeta$ for scale parameter $x=500$. The dashed lines show the formulas found in the literature and solid lines correspond to approximations introduced in the present article: a Green \& Barnum approximation (Green and Barnum 1963), b Fitzmaurice approximation (Fitzmaurice 1964), c Swider approximation (Swider 1964), d Titheridge empirical formula (Titheridge 1988), e Kocifaj formula (Kocifaj 1996), f Huestis asymptotics, 1 term (Huestis 2001), g present work, Eq. (35), h present work, Eq. (36) 
asymptotic expansions and is valid for all values of zenith angle and is simpler to implement in practice.

To conclude this section we provide an explicit expression for approximation (36) in the form useful for numeric calculations
(1) and $\mathrm{Ch}^{(a)}(X, \zeta)$ is the analytical approximation of this function calculated with the double precision.

We use the relative error instead of the absolute error $\varepsilon_{a}=\left|\mathrm{Ch}^{(e)}-\mathrm{Ch}^{(a)}\right|$ due to the following reasoning. For applications in atmospheric physics the values of

$$
\begin{aligned}
\operatorname{Ch}(X, \zeta) \approx & \sqrt{\frac{\pi}{2}} \sqrt{X \sin \zeta}\left(1+\frac{3}{8} \frac{1}{X \sin \zeta}\right) e^{X(1-\sin \zeta)} \operatorname{erfc}[\sqrt{X(1-\sin \zeta)}] \\
& +\frac{1}{\sqrt{1-\sin ^{2} \zeta}}\left\{1-t-\frac{1}{X \sin \zeta\left(1-\sin ^{2} \zeta\right)^{2}}\right. \\
& {\left.\left[\sin ^{3} \zeta-t^{3}+\frac{3}{8} t\left(1-\sin ^{2} \zeta\right)\right]\right\}, } \\
t= & \sqrt{\sin \zeta(1+\sin \zeta) / 2} .
\end{aligned}
$$

Here the zenith angle enters as an argument of sine function only and the expression $\sin \zeta$ is optimal to be evaluated prior the substitution in (37). Finally we note that for sufficiently large values of $X$ as $\zeta \rightarrow 0$ the formula (37) approaches the expected limit $\sec \zeta$.

\section{Methodology of comparison}

Fortran code was written to evaluate $\mathrm{Ch}(X, \zeta)$ through the analytical approximations (35), (36) and (43). These equations has adopted in the form optimal for numerical calculations, cf. Eq. (37). For evaluation of the numerical integral (1) and some of the previous analytical approximations of Table 1 the code written by Huestis (2001) has been adopted. We have chosen the double precision calculations for both approximative formulas and numerical integral (1) to minimize the effect of rounding errors. The code was compiled and tested using the Intel Fortran Compiler under Windows operating system. Based on the Fortran program the Python package has been created using F2Py Fortran-Python interface generator and has been implemented as the corresponding wrapper program.

In order to show the precision of various analytical approximations we plotted the logarithm of the relative error as a function of the solar zenith angle in Figs. 1 and 2 for scale parameters $X=50$ and $X=500$, respectively. The choice of the scale parameters is arbitrary and chosen to satisfy $X \gg 1$ and to represent the values different in one order of magnitude. The relative error is defined as

$$
\varepsilon=\frac{\left|\mathrm{Ch}^{(e)}(X, \zeta)-\mathrm{Ch}^{(a)}(X, \zeta)\right|}{\mathrm{Ch}^{(e)}(X, \zeta)},
$$

where $\mathrm{Ch}^{(e)}(X, \zeta)$ is the exact value of the Chapman function taken from the double precision numerical integral the scale parameter that satisfy $X \gg 1$ are of interest. For $\zeta \in[0, \pi / 2]$ in this asymptotic one can show that $\mathrm{Ch}^{(e)}=O(\sqrt{X})$ and $\mathrm{Ch}^{(a)}=O(\sqrt{X})$, where $O(x)$ is the order symbol (Erdélyi 2003). These relations follows from Eq. (5) and from the asymptotic expansion of the Macdonald function. In order to remove the $O(\sqrt{X})$ trend in the error analysis the relative error has been chosen. Similar approach has been chosen by D. L. Huestis in his thorough analysis of Chapman function evaluation (Huestis 2001). The current error analysis is thus fully in agreement with those performed in Ref. Huestis (2001) apart from the fact that D. L. Huestis chosen the scale parameters $X=50$ and $X=800$ and we use $X=50$ and $X=500$ in the current study. We have also checked the correspondence of several approximative formulas from Table 1 with the error analysis of corresponding expressions in Ref. Swider and Gardner (1967) for $X=50$ and

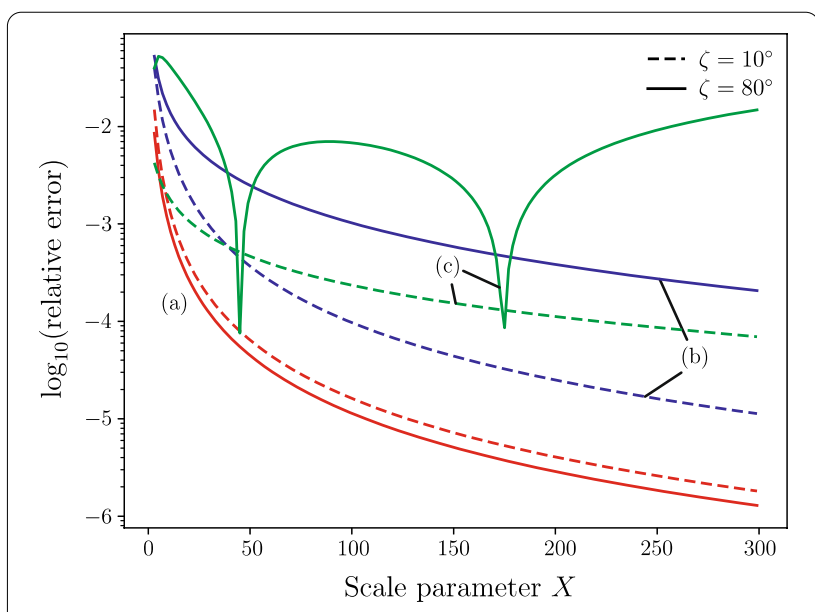

Fig. 3 Relative error as a function of the scale parameter for small (dashed) and large (solid) solar zenith angles. Three analytical formulas are compared: $\mathbf{a}$ is Eq. (36) obtained in this work, $\mathbf{b}$ is the Kocifaj formula (Kocifaj 1996) that shows the best performance among analytical approximations found in the literature, and $\mathbf{c}$ is the empirical formula of Green and Barnum (1963); Green et al. (1964) 
$X=500$. A good agreement has been found despite of difference in some computational aspects.

As has been already mentioned, the majority of analytic approximations for the Chapman function involve the complimentary error function. For all such expressions we have used the specific rational approximations for this function due to Smith and Smith (1972)

$$
\operatorname{erfc}(x) \approx \begin{cases}\frac{1.0606963+0.55643831 x}{1.0619896+1.724569 x+x^{2}} e^{-x^{2}}, & 0 \leq x \leq 8, \\ \frac{0.56498823}{0.06651874+x} e^{-x^{2}}, & 8<x \leq 100 .\end{cases}
$$

In this way we reduced the approximation of Chapman function due to Smith \& Smith to the Fitzmaurice approximation (Fitzmaurice 1964). We note that the approximative formula (39) originates from the tables of approximations collected in Ref. Hart (1968) and yields the absolute error in double precision calculations of orders $10^{-5}$ and $10^{-3}$ for $x \in[0,8]$ and $x \in(8,100]$, respectively.

\section{Results of comparison and discussion}

The plotted in Figs. 1, 2 and 3 relative errors (38) for the analytical approximations of Chapman function [see Table 1 and Eqs. (35), (36)] exhibit different performance. For example, the Green \& Barnum empirical formula shows the pure performance for zenith angles $\zeta \rightarrow \pi / 2$ as has also been noted in Huestis (2001), see Figs. 2(a) and $3(\mathrm{c})$. The Fitmaurice formula reaches the discrepancy of several percents from the numerically obtained values at moderate vales of $X$, e.g. around $1 \%$ at $X=50$, see Fig. 1(b). Due to its analytical simplicity this function gained the popularity in modeling of the atmosphere. For example, the Thermosphere Ionosphere Electrodynamics General Circulation Model (Dickinson et al. 1981) or the Gauss-Seidel limb scattering radiative transfer model (Loughman et al. 2015) use the Fitzmaurice formula and hence it also incorporates these discrepancies from the exact formula. With this respect it is worth to point that the Kocifaj formula (curve (e) in 1,2) has an attractive property of being quite accurate and analytically simple. As one can see this formula, remaining rather unknown to atmospheric physics community, performs even better than the highly-accurate formula of Swider [curve (c)] or the approximation due to Huestis [curve (f)].

Another accurate analytic approximation of the Chapman integral is the semi-empirical formula of Titheridge introduced in Titheridge (1988) and corrected in Titheridge (2000), see curve (d). The discrepancy of this approximation from the Chapman function grows however for large values of $X$ and when $\zeta \rightarrow \pi / 2$ as can be seen in Fig. 2.

Two solid curves shown in Figs. 1 and 2 were obtained in the present work using the established relations (15) and (27). As has been shown in the previous section the asymptotic expansion of Eq. (15), cf. curve (g), reduces to the zeroth-order term in uniform asymptotic expansion of Eq. (27), cf. curve (h). Still, being only the first term given by (35), it outperforms the analytical approximations of Fitzmaurice [curve(b)] and the first-term of asymptotic expansion of Huestis [curve (f)]. The analytical approximation (36), curve (h), shows the best performance among the analytical approximations. Oscillatory behavior of this curve arises from variation in accuracy of polynomial approximations to the mathematical function $\operatorname{erfc}(x)$ and of the expression $\sqrt{\pi / 2 x}(1+3 / 8 x) \exp (-x)$ being the asymptotic expansion of Bessel function $K_{1}(x)$.

In order to illustrate the dependence of the obtained approximation formula (36) on the scale parameter, we compare it with the analytical approximation of Kocifaj and Green \& Barnum in Fig. 3. The curve (a) calculated with Eq. (36) performs better than the Kocifaj formula [curve (b)] or Green \& Barnum formula [curve (c)] for all relevant scale parameters values in atmospheric applications. It is worth to note that in some applications (e.g., radiative transfer and radiometric sounding), where the Chapman mapping function is used as a core function inside multiple integrals, the complexity of derived analytical approximations (35), (36) will require additional computational resources and analytically simpler and accurate Kocifaj's approximation is preferable. Finally, we remark that for small values of the scale parameter the approximation (43) of Appendix A performs better than other analytical approximations, however this case is less interesting for known application cases.

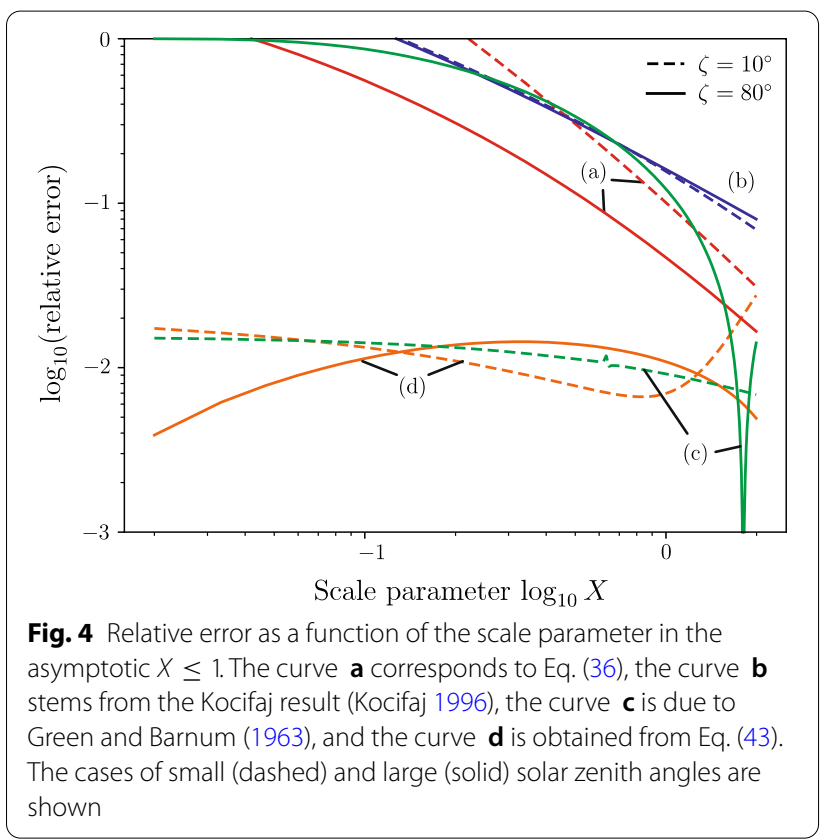




\section{Conclusions}

We have shown the relationship of the Chapman function, $\mathrm{Ch}(X, \zeta)$, to several families of the incomplete Bessel functions. This allowed us to derive the accurate analytical approximation of $\mathrm{Ch}(X, \zeta)$ for values of the scale parameter $X$ and the solar zenith angle $\zeta$ relevant for calculations of atmospheric attenuation, ionospheric production rates, optical masses, etc. for a model exponential atmosphere. For all values of $X$ and $\zeta$ the obtained analytical approximation provides the best accuracy among other known approximations found in the literature.

Despite the Chapman function has been derived under certain idealized assumptions for the atmosphere to be exponential, non-refractive, and spherically symmetric, its introduction allowed one to depart from the very simplified model of a plan-parallel atmosphere. In many practical situations one can split the real atmosphere into spherical layers, e.g., by studying the atmospheric refraction, or one can consider the atmospheric constituents separately, e.g., by studying the photoionization of the ionosphere. By doing this it is possible to approximate the realistic atmospheres with the set of idealized ones. In this respect the Chapman mapping integral or its analytic approximations remain a useful tool in modeling. We thus believe that the given overview of existing approximations of the Chapman function as well as the obtained serve merely for providing the complete picture on analytic approximations for the Chapman function.

The proposed approximation is based on Eq. (31) which relates the Chapman function and the incomplete Macdonald function (13) and the the incomplete Weber integral (28). The former function can be approximated as shown in Eq. (32), while for the incomplete Weber integral we use the following approximation

$$
\begin{aligned}
& \widetilde{Q}_{0}\left(X \sin ^{2} \frac{\zeta}{2}, X \sin \zeta\right) \approx \\
& e^{2 X \sin ^{2} \frac{\zeta}{2}}\left(1-e^{-X \cos ^{2} \frac{\zeta}{2}}\right) \\
& \quad \times \exp \left\{-\left[2 \mu\left(2 X \cos ^{2} \frac{\zeta}{2}\right) \tan \frac{\zeta}{2}\right]^{\lambda\left(2 X \cos ^{2} \frac{\zeta}{2}\right)}\right\},
\end{aligned}
$$

which derivation and discussion has been performed in Ref. Vasylyev et al. (2013). Here

$$
\lambda(x)=4 x \frac{e^{-x} I_{1}(x)}{1-e^{-x} I_{0}(x)}\left\{\ln \left[2 \frac{1-e^{-x / 2}}{1-e^{-x} I_{0}(x)}\right]\right\}^{-1}
$$

and

$$
\mu(x)=\left\{\ln \left[2 \frac{1-e^{-x / 2}}{1-e^{-x} I_{0}(x)}\right]\right\}^{1 / \lambda(x)}
$$

are the shape and scale functions expressed in terms of the modified Bessel functions $I_{n}(x)$. Substituting (40) in (31) we obtain the Chapman function approximation

$$
\begin{aligned}
\operatorname{Ch}(X, \zeta) \approx & X \sin \zeta e^{X}\left\{K_{1}(X \sin \zeta)+\frac{I_{1}(X \sin \zeta)}{I_{0}(X \sin \zeta)}\left[K_{0}(X \sin \zeta)-K_{0}(X \sin \zeta, \beta)\right]\right. \\
& -\left[K_{1}(X \sin \zeta)+\frac{I_{1}(X \sin \zeta)}{I_{0}(X \sin \zeta)} K_{0}(X \sin \zeta)\right] \\
& \times\left(2\left(1-e^{-X \cos ^{2} \frac{\zeta}{2}}\right) \exp \left\{-\left[2 \mu\left(2 X \cos ^{2} \frac{\zeta}{2}\right) \tan \frac{\zeta}{2}\right]^{\lambda\left(2 X \cos ^{2} \frac{\zeta}{2}\right)}\right\}\right. \\
& \left.\left.-\left[1-e^{-X} I_{0}(X \sin \zeta)\right]\right)\right\}
\end{aligned}
$$

new results would be of interest in the context of realistic atmospheres modeling.

\section{Appendix A}

In this appendix we provide the analytical approximation of the Chapman function for the case $X \leq 1$. Such small values of the scale parameter are not relevant for applications in atmospheric physics and the obtained formulas
Figure 4 shows the performance of the obtained approximation as a function of the scale parameter for $X \leq 1$. Despite the obtained approximation has a discrepancy of several percent with the exact numerical integral, its performance is still better for considered range of $X$ parameter than other considered approximative formulas. We also note that the accuracy of the approximation can be improved with the procedure developed in Ref. Vasylyev et al. (2013). 


\section{Acknowledgements}

The author gratefully acknowledges J. Berdermann and E. Schmölter for fruitful discussions.

\section{Authors' contribution}

DV derived the relationships with the families of the incomplete Bessel functions, proposed the new analytical approximation formulas for the Chapman integral, wrote the Fortran code and the corresponding Python wrapper for calculation of the introduced analytic approximations and prepared all plots. The author read and approved the final manuscript.

\section{Funding}

The work was carried out within the programmatic funding of the German Aerospace Center.

\section{Availability of data and materials}

For numerical calculations used in this article we adopted and extended the original Fortran code by Huestis (2001). The calculation of special functions was performed using the algorithms according to Press et al. (1992), which were adapted for double precision calculations. The Fortran code (Fortran 90) for calculation of Chapman function and its analytic approximations in different forms is available along its Python wrapper (Python 3.8) at web address https://gitlab.com/heidima/chapmanfunction. The Fortran code compilation has been performed via the Intel Fortran Compiler.

\section{Declarations}

\section{Competing interests}

The author declares that he has no competing interests.

Received: 22 February 2021 Accepted: 6 May 2021

Published online: 21 May 2021

\section{References}

Abramowitz M, Stegun IA (1972) Handbook of Mathematical Functions with Formulas, Graphs, and Mathematical Tables. Wiley, New York

Agrest MM, Maximov MS (1971) Theory of Incomplete Cylindrical Functions and Their Applications. Springer, Berlin

Brasseur GP, Solomon S (2005) Aeronomy of the Middle Atmosphere. Springer, Berlin

Chapman S (1931) The absorption and dissociative or ionizing effect of monochromatic radiation in an atmosphere on a rotating earth part II. Grazing incidence. Proc Phy Soc 43(5):483-501

Chapman S (1953) Note on the grazing-incidence integral ch $(x, x)$ for monochromatic absorption in an exponential atmosphere. Proceed Physical Soc Sect B 66(8):710-712. https://doi.org/10.1088/0370-1301/66/8/411

Dahlback A, Stamnes K (1991) A new spherical model for computing the radiation field available for photolysis and heating at twilight. Planetary Space Sci 39(5):671-683. https://doi.org/10.1016/0032-0633(91)90061-E

Dickinson RE, Ridley EC, Roble RG (1981) A three-dimensional general circulation model of the thermosphere. J Geophy Res Space Phy 86(A3):14991512. https://doi.org/10.1029/JA086iA03p01499

Erdélyi A (2003) Asymptotic Expansions. Dover, New York

Extensive Grieder P, Showers Air (2010) High Energy Phenomenaand Astrophysical Aspects, vol 1. Springer, Berlin

Fitzmaurice JA (1964) Simplification of the Chapman function for atmospheric attenuation. Appl Opt 3(5):640-640. https://doi.org/10.1364/AO.3.000640

Friedrich M, Torkar KM (2001) Firi: A semiempirical model of the lower ionosphere. J Geophys Res Space Phys 106(A10):21409-21418. https://doi. org/10.1029/2001JA900070

Green AES, Barnum L (1963) Note on a simple approximation to the chapman function. Space Sci. Lab., General Dynamics/Astronautics, San Diego, California, Technical report

Green AES, Lindenmeyer CS, Griggs M (1964) Molecular absorption in planetary atmospheres. J Geophys Res 69(3):493-504. https://doi.org/10. 1029/JZ069i003p00493

Hart JF et al (1968) Computer Approximations. John Wiley \& Sons, New York
Huestis DL (2001) Accurate evaluation of the Chapman function for atmospheric attenuation. J Quantit Spectrosc Radiat Transf 69(6):709-721. https://doi.org/10.1016/S0022-4073(00)00107-2

Jones DS (2007) Incomplete Bessel functions. I. Proceedings of the Edinburgh Mathematical Society 50(1), 173-183. https://doi.org/10.1017/S0013 091505000490

Kocifaj M (1996) Optical air mass and refraction in a Rayleigh atmosphere. Contrib Astron Obs Skalnate Pleso 26:23-30

Loughman R, Flittner D, Nyaku E, Bhartia PK (2015) Gauss-Seidel limb scattering (GSLS) radiative transfer model development in support of the Ozone Mapping and Profiler Suite (OMPS) limb profiler mission. Atmo Chem Phy 15(6):3007-3020. https://doi.org/10.5194/acp-15-3007-2015

Press WH, Teukolsky SA, Vetterling WT, Flannery BP (1992) Numerical Recipes in Fortran. The Art of Scientific Computing. Cambridge University Press, Cambridge

Rapp-Arrarás, I., Domingo-Santos, J.M.: Functional forms for approximating the relative optical air mass. Journal of Geophysical Research: Atmospheres 116(D24) (2011). https://doi.org/10.1029/2011JD016706

Schüler C (2012) An approximation to the chapman grazing-incidence function for the atmospheric scattering. In: Engel, W. (ed.) GPU Pro ${ }^{3}$ : Advanced Rendering Technique, pp. 105-118. CRC Press, Boca Raton (2012). Chap. 2

Schunk R, Nagy A (2009) Ionospheres. Physics, Plasma Physics, and Chemistry. Cambridge University Press, Cambridge

Smith FL III, Smith C (1972) Numerical evaluation of Chapman's grazing incidence integral $c h(x, \chi)$. J Geophys Res 77(19):3592-3597. https://doi.org/ 10.1029/JA077i019p03592

Swider W (1964) The determination of the optical depth at large solar zenith distances. Planet Space Sci 12(8):761-782. https://doi.org/10.1016/00320633(64)90056-X

Swider W, Gardner ME (1967) On the accuracy of certain approximations for the Chapman function. Report AFCRL-67-0468. Technical report, L. G. Hanscom Field, Air Force Cambridge Research Laboratory, Bedford, Massachusetts

Terras R (1981) A Miller algorithm for an incomplete bessel function. J Comput Phys 39(1):233-240. https://doi.org/10.1016/0021-9991(81)90147-9

Titheridge JE (1988) An approximate form for the Chapman grazing incidence function. J Atmos Terrestr Phys 50(8):699-701. https://doi.org/10.1016/ 0021-9169(88)90033-5

Titheridge JE (2000) Modelling the peak of the ionospheric E-layer. J Atmos Solar Terrestr Phys 62(2):93-114. https://doi.org/10.1016/S1364-6826(99) 00102-9

Vasylyev DY, Semenov AA, Vogel W (2013) Quantum channels with beam wandering: an analysis of the Marcum Q-function. Physica Scripta T153:014062. https://doi.org/10.1088/0031-8949/2013/t153/014062

Velinov P (1974) Cosmic ray ionization rates in the planetary atmospheres. J Atmos Terrestr Phys 36(2):359-362. https://doi.org/10.1016/00219169(74)90055-5

Wallach D, Hapke B (1985) Light scattering in a spherical, exponential atmosphere, with applications to Venus. Icarus 63(3):354-373. https://doi.org/ 10.1016/0019-1035(85)90052-1

\section{Submit your manuscript to a SpringerOpen ${ }^{\circ}$ journal and benefit from:}

- Convenient online submission

- Rigorous peer review

- Open access: articles freely available online

- High visibility within the field

- Retaining the copyright to your article

Submit your next manuscript at springeropen.com 